\title{
Experimental Assessment of the Performance of an Electromagnetic Hearing Aid in Human Temporal Bones*
}

\author{
Shinji HAMANISHI**, Takuji KOIKE***, Wade CHIEN ${ }^{* * * *}$, \\ Michael E. RAVICZ****, John J. ROSOWSKI****, \\ Saumil N. MERCHANT ${ }^{* * * *}$, Toshimitsu KOBAYASHI ${ }^{\dagger}$ and Hiroshi WADA**
}

\begin{abstract}
To circumvent some of the problems inherent in conventional hearing aids such as low gain at high frequencies due to acoustic feedback, discomfort in occlusion of the external ear canal and so on, implantable hearing aids have been developed over the past two decades. The most prominent feature of implantable hearing aids is that a transducer is directly coupled to the one of the middle-ear ossicles. However, since invasive surgery is necessary for implantation of these hearing aids, they have not as yet been widely employed. We therefore constructed a prototype of a non-implantable hearing aid which is mainly composed of a microphone amplifier system and an electromagnetic transducer developed in our previous study. It can generate an excitation force to vibrate the ossicles by a coil adhered to the tympanic membrane. In this study, the excitation force generated by this hearing aid was evaluated using human temporal bones. The best result of experiments using three bones indicates that the newly developed hearing aid can generate an excitation force of more than $80 \mathrm{~dB}$ SPL in terms of sound pressure at frequencies between 0.8 and $3.2 \mathrm{kHz}$.
\end{abstract}

Key Words: Electromagnetic, Hearing Aid, Middle Ear, Tympanic Membrane, Human Temporal Bone

\section{Introduction}

Hearing impairment is the one of the most common physical disabilities. In Japan, there are more than six million individuals (nearly $5 \%$ of population) with hearing loss. In the case of the United States, more than twentyfive million individuals (nearly $9 \%$ of population) are impaired. Most such individuals suffer from sensorineural hearing loss, which arises from damage in the inner ear. The loss mainly occurs in the higher frequency range and such patients have difficulties in speech discrimination.

* Received 7th July, 2005 (No. 05-4089)

** Department of Bioengineering and Robotics, Tohoku University, 6-6-01 Aoba-yama, Sendai 980-8579, Japan.

E-mail: hama@wadalab.mech.tohoku.ac.jp

*** Department of Mechanical Engineering and Intelligent Systems, The University of Electro-Communications, 15-1 Chofugaoka, Chofu, Tokyo 182-8585, Japan

**** Eaton-Peabody Laboratory, Massachusetts Eye and Ear Infirmary, Boston, MA 02114, U.S.A.

$\doteqdot$ Department of Otorhinolaryngology - Head and Neck Surgery, Tohoku University, 1-1 Seiryo-machi, Sendai 980-8574, Japan
Although the majority of these individuals can be treated with conventional hearing aids, which are mainly composed of a microphone, an amplifier, and an earphone, some patients who use conventional hearing aids remain unsatisfied owing to various inherent problems. For example, the proximity of the microphone to the earphone causes acoustic feedback, i.e., the output from the earphone is picked up by the microphone and amplified repeatedly. This is one of the reasons why it is difficult to obtain high acoustic gain with conventional hearing aids. Furthermore, conventional hearing aids occlude the external ear canal and this occlusion causes discomfort for hearing-aid wearers.

Development over the past two decades has shown that implantable hearing aids can circumvent some of problems found in conventional hearing aids. The most prominent feature of implantable hearing aids is that a transducer is directly coupled to the one of the middleear ossicles. With such hearing aids, there are two major methods for the achieving excitation of the ossicles, i.e., by piezoelectric transducers and by electromagnetic transducers. Suzuki et al. ${ }^{(1)}$ and Gyo et al. ${ }^{(2)}$ developed a piezoelectric transducer. It is a piezoelectric ceramic bi- 
morph, which is attached to the stapes to vibrate it. Vibrant Soundbridge ${ }^{\circledR}$, on the other hand, is commercially available electromagnetic hearing aid ${ }^{(3)}$. Its transducer is composed of a magnet surrounded by two induction coils and is coupled to the incus to vibrate it directly. Besides the above-mentioned devices, many other types of piezoelectric and electromagnetic transducers have been developed $^{(4)-(13)}$. However, implantable transducers are not as yet widely used because invasive surgery is necessary. Thus, it is difficult to apply them in children who are still growing.

In our previous study ${ }^{(14)}$, we constructed a highly efficient electromagnetic transducer, which is composed of three coils and a magnet. One of the coils is adhered to the tympanic membrane and vibrated by electromagnetic force, which acts between the magnet fixed in the external ear canal and the coil. Experiments using an artificial middle ear, which imitated the human tympanic membrane and ossicles, showed that a prototype of this optimally designed electromagnetic transducer was able to generate an excitation force of more than $95 \mathrm{~dB}$ in terms of sound pressure at frequencies between 0.1 and $10 \mathrm{kHz}$. In addition, in order to assess the transducer's performance from the point of view of physiological response, the cochlear microphonic (CM), which arises from sum of electrical potentials in the hair cells of the cochlea, was measured in anesthetized guinea pigs. This experiment showed that the transducer generated an excitation force of $93-106 \mathrm{~dB}$ SPL in terms sound pressure at frequencies between 0.5 and $10 \mathrm{kHz}$.

The suitability of the electromagnetic hearing aid for use in humans, however, is uncertain because frequency characteristics of ears in humans are different from those in artificial middle ears and guinea pigs. Experiments in human ears are therefore required to assess the performance of this hearing aid. In this study, we firstly constructed a prototype of a non-implantable hearing aid by combining the electromagnetic transducer previously developed with a microphone amplifier system. The excitation force generated by this hearing aid was then evaluated using human temporal bones.

\section{Non-Implantable Electromagnetic Hearing Aid}

An example of application of a non-implantable electromagnetic hearing aid to the human ear is shown in Fig. 1 (a) ${ }^{(14)}$. It consists of two parts, i.e., a microphone amplifier system and an electromagnetic transducer. The electromagnetic transducer was designed to enable its placement in the human external ear canal.

Figure 1 (b) shows a schematic of the electromagnetic transducer. It is composed of a core, driving and induction coils, a rare-earth magnet and a vibrator coil. Co-based amorphous magnetic material with high permeability was used for the core. The diameter and length of the core are

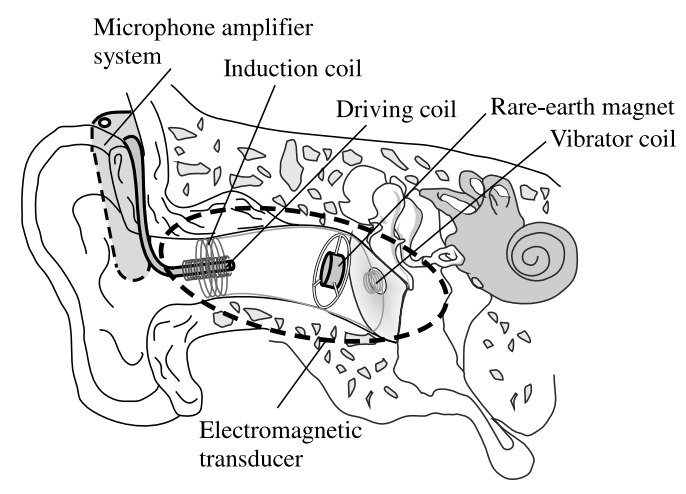

(a)

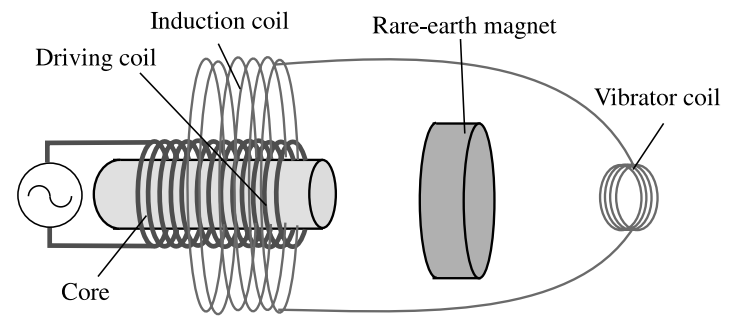

(b)

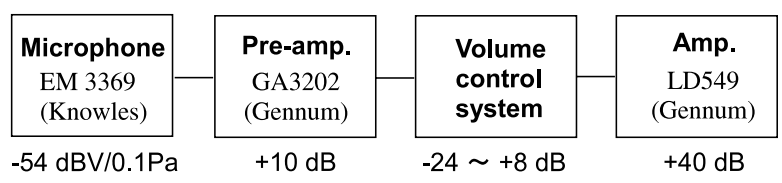

(c)

Fig. 1 Non-implantable electromagnetic hearing aid. (a) Image of the application of the non-implantable hearing aid in the human. The microphone amplifier system, the core and the driving coil are easy to put on and remove. (b) Schematic of the electromagnetic transducer. Co-based amorphous magnetic material with high permeability is used for the core material and the driving coil is wound around it. A cylindrical-shaped Ne-Fe-B magnet is used. The vibrator coil is attached to a plastic plate, which is in contact with the tympanic membrane. Total mass of the vibrator coil and plastic plate is $18.3 \mathrm{mg}$. (c) Block diagram of the microphone amplifier system of the hearing aid. This system is composed of a microphone (EM3369, Knowles), a preamplifier (GA3202, Gennum), a volume control system and a main amplifier (LD549, Gennum).

$2 \mathrm{~mm}$ and $30 \mathrm{~mm}$, respectively. The driving coil contains 2320 turns and is wound with insulated copper wire $60 \mu \mathrm{m}$ in diameter. Its inner diameter and length are the same as those of the core and its outer diameter is $2.8 \mathrm{~mm}$. The induction coil, which is placed in the external ear canal, contains 100 turns of the same wire as that used for the driving core coil. Its inner and outer diameters are $4 \mathrm{~mm}$ and $6 \mathrm{~mm}$, respectively, and its length is $4 \mathrm{~mm}$. The permanent magnet used in this study is a cylindrical shaped $\mathrm{Ne}-\mathrm{Fe}-\mathrm{B}$ magnet with a surface magnetic density of $1.2 \mathrm{~T}$ and is fixed by a holder near the tympanic membrane. The 
diameter and thickness of the magnet are $3 \mathrm{~mm}$ and $2 \mathrm{~mm}$, respectively. The disk-shaped vibrator coil contains 14 turns and is wound with insulated copper wire $60 \mu \mathrm{m}$ in diameter. Its inner and outer diameters are $1.3 \mathrm{~mm}$ and $2.5 \mathrm{~mm}$, respectively. The vibrator coil is glued to a plastic plate, which is used for the attachment to the tympanic membrane. This plastic plate with a diameter of $1.5 \mathrm{~mm}$ is of conical shape and designed so as to fit onto the surface of the tympanic membrane at the umbo by means of oil. The total mass of the vibrator coil and plastic plate is $18.3 \mathrm{mg}$.

When an alternating current is supplied to the driving coil, magnetic flux passes through it and a current is induced in the induction coil. The induced current that flows into the vibrator coil causes magnetic interaction between the magnetic field generated by the vibrator coil and the static magnetic flux of the magnet. As a result, the vibrator coil, which is in contact with the tympanic membrane, is vibrated by the repulsive and attractive forces between the magnet and the vibrator coil.

Figure 1 (c) shows a block diagram of the microphone amplifier system of the hearing aid. This system consists of four parts, i.e., a microphone (EM3369, Knowles), a preamplifier (GA3202, Gennum), a volume control system and a main amplifier (LD549, Gennum). The sensitivity of the microphone is $-54 \mathrm{dBV} / 0.1 \mathrm{~Pa}$ at $1.0 \mathrm{kHz}$. Gain of the preamplifier and that of the main amplifier are +10 and $+40 \mathrm{~dB}$ at $1.0 \mathrm{kHz}$, respectively. The gain can be changed by the volume control system in the range between -24 and $+8 \mathrm{~dB}$. The minimum and maximum values of total gain in this microphone amplifier system are therefore +26 and $+58 \mathrm{~dB}$, respectively. For example, when the gain of the volume is maximum $(+8 \mathrm{~dB})$, an acoustic stimulus of $74 \mathrm{~dB} \operatorname{SPL}(0.1 \mathrm{~Pa})$ at $1.0 \mathrm{kHz}$, is detected by the microphone, is firstly transformed into an electrical signal of $-54 \mathrm{dBV}\left(2.0 \times 10^{-3} \mathrm{~V}\right)$. This signal is then amplified by $+58 \mathrm{~dB}$. Output voltage, which is applied to the driving coil, is therefore $+4 \mathrm{dBV}(1.58 \mathrm{~V})$.

\section{Methods}

The fundamental properties of the hearing aid were evaluated using three human temporal bones, namely, Bone \#1 (63-year-old male), Bone \#2 (53-year-old female) and Bone \#3 (46-year-old male). They were extracted from the cadavers within $32 \mathrm{~h}$ of death. The tympanic membrane and the middle ear were inspected using an operating microscope. Simple mastoidectomy and posterior hypotympanotomy were performed and the pinna, the external ear canal, some of the mastoid portion of the facial nerve and surrounding bone were removed for a clear and wide view of the stapes. The temporal bone was then secured in a temporal bone holder. The tympanic membrane, ossicles, suspensory ligaments, tensor tympani and stapedius muscle were left intact during the experiments.

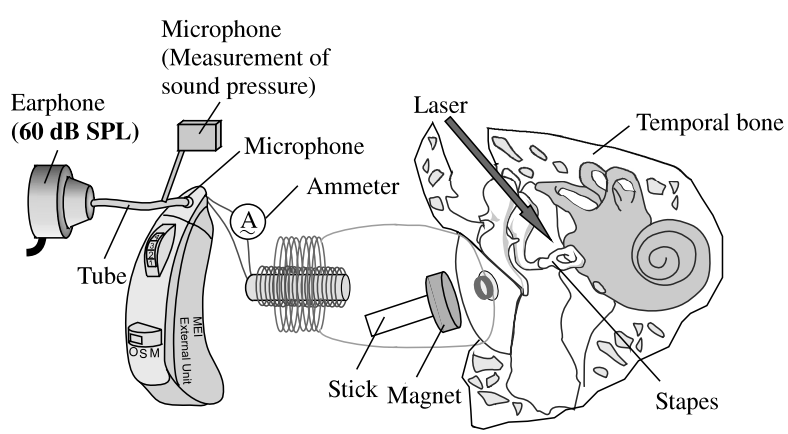

(a)

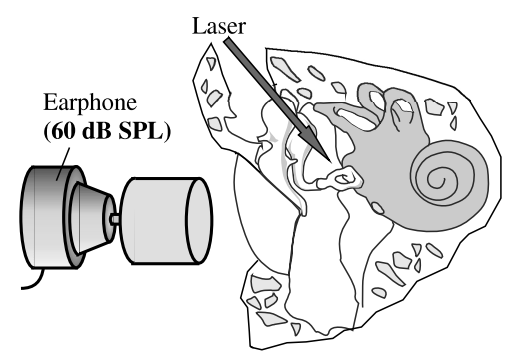

(b)

Fig. 2 Experimental set up for measuring the frequency responses of the posterior crus of the stapes. (a) Excitation of the tympanic membrane by the electromagnetic hearing aid. (b) Excitation of the tympanic membrane by acoustical stimulation. The distance between the magnet and the vibrator coil was set at 1 and $3 \mathrm{~mm}$.

The vibrator coil was adhered to the tympanic membrane with oil as shown in Fig. 2 (a). As the pinna and the external ear canal had already been removed, the microphone amplifier system, the core, and the driving and induction coils were placed on a stage, which was $0.15 \mathrm{~m}$ apart from the temporal bone. The induction and vibrator coils were connected by a copper wire $0.3 \mathrm{~m}$ in length. The magnet was held by a stick holder and the distance between the magnet and the vibrator coil was set at 1 and $3 \mathrm{~mm}$.

As shown in Fig. 2 (a), in order to apply sound pressure to the microphone of the hearing aid, an earphone (ER-4C, Etymotic Research) was connected to the microphone of the hearing aid via a 3-cm-long vinyl tube. In addition, another microphone (EK3027, Knowles) was placed $1 \mathrm{~cm}$ apart from the microphone of the hearing aid for monitoring of the constant sound pressure.

Frequency responses of the posterior crus of the stapes were then measured with a laser Doppler velocimeter (HLV-1000, Polytec) when a constant sound pressure of $60 \mathrm{~dB}$ SPL with a sweep frequency from 0.2 to $10 \mathrm{kHz}$ was applied to the microphone of the hearing aid by the earphone. During the experiments, the current which flows to the driving coil was also measured with an ammeter which was connected between the microphone amplifier system and driving coil. Next, the hearing aid was 
removed as shown in Fig. 2 (b) and the frequency response of posterior crus of the stapes was measured again when the same acoustic stimulation as that in the case of the hearing aid was directly applied to the tympanic membrane by the earphone. All experiments were performed at the Massachusetts Eye and Ear Infirmary, Boston, MA, U.S.A.

\section{Results}

\subsection{Frequency responses of the microphone and amplifier system}

Figure 3 shows the frequency response of the current, which flows to the driving coil, and that of the sensitivity of the microphone. The current is almost constant up to $2.0 \mathrm{kHz}$. From $2.0 \mathrm{kHz}$, the current increases with an increase in the frequency and the maximum current is $23 \mathrm{~dB}$ re $1 \mathrm{~mA}$ at $5.0 \mathrm{kHz}$. This value is $15 \mathrm{~dB}$ higher than that at $2.0 \mathrm{kHz}$. Above $5.0 \mathrm{kHz}$, the frequency response of the current has a notch at $6.5 \mathrm{kHz}$ and peaks at $8.0 \mathrm{kHz}$. The sensitivity of the microphone increases with an increase in the frequency up to $8.0 \mathrm{kHz}$, where the sensitivity is $-42 \mathrm{~dB}$ re $1 \mathrm{~V} / 0.1 \mathrm{~Pa}$. From $8.0 \mathrm{kHz}$, the sensitivity decreases.

As shown in this figure, above $2.0 \mathrm{kHz}$, the tendency of the frequency response of the current is similar to that of the sensitivity of the microphone, i.e., the current increases with an increase in the sensitivity of the microphone. Such increase in the current at high frequencies is caused by the increase in the output voltage in the microphone due to the increase of its sensitivity. However, the current measured at $4.0 \mathrm{kHz}$ is approximately $10 \mathrm{~dB}$ greater than that expected from the sensitivity of the microphone. The gain of the preamplifier increases by $3 \mathrm{~dB}$ with an increase in frequency from 0.1 to $10 \mathrm{kHz}$. Regarding the applied sound pressure level, its practical value at the microphone of the hearing aid may possibly be greater than $60 \mathrm{~dB}$ as the sound pressure was measured at a point

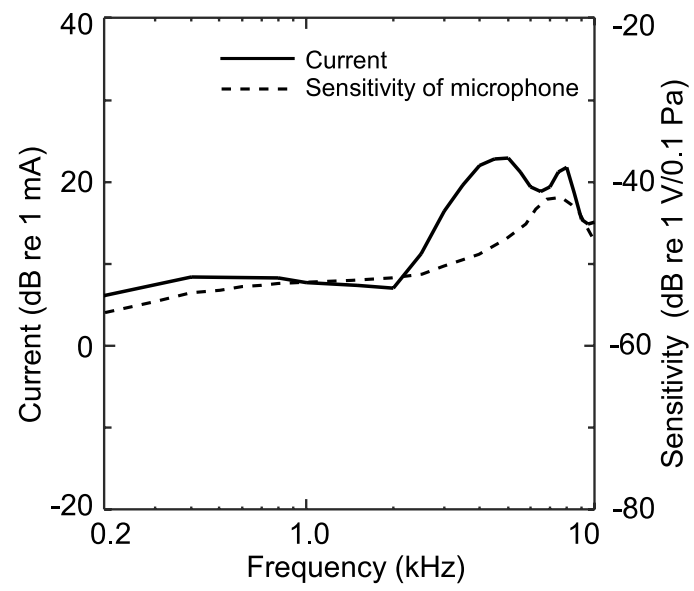

Fig. 3 Frequency responses of the current, which flows to the driving coil and the sensitivity of the microphone.
$1 \mathrm{~cm}$ from the microphone. These two factors may possibly cause the measured current at $4.0 \mathrm{kHz}$ to increase.

\subsection{Frequency responses of the stapes}

The frequency responses of the posterior crus of the stapes are shown in Fig. 4. The regions of the measurement data for Bones \#1, \#2 and \#3 which contain noise at low frequencies are shown by gray lines. The measurable frequency range is therefore restricted to the region

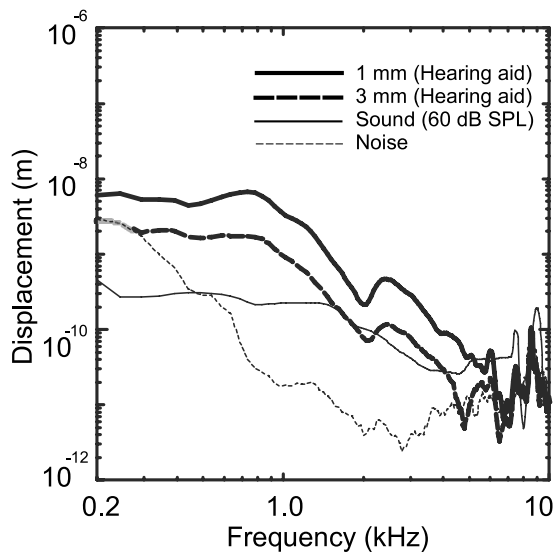

(a)

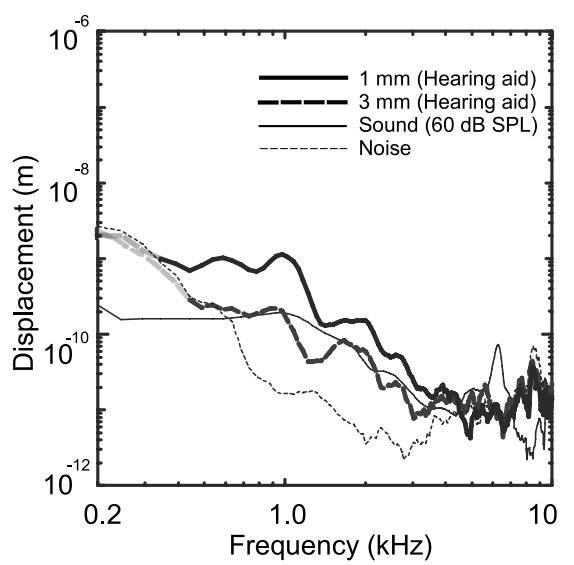

(b)

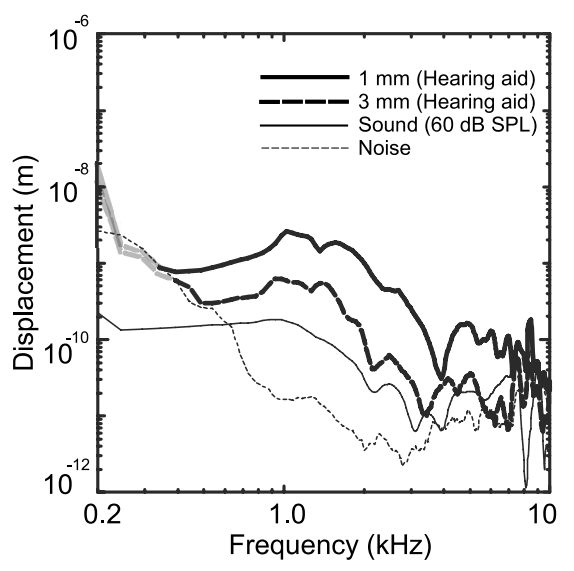

(c)

Fig. 4 Frequency responses of the posterior crus of the stapes. (a) Bone \#1. (b) Bone \#2. (c) Bone \#3. 


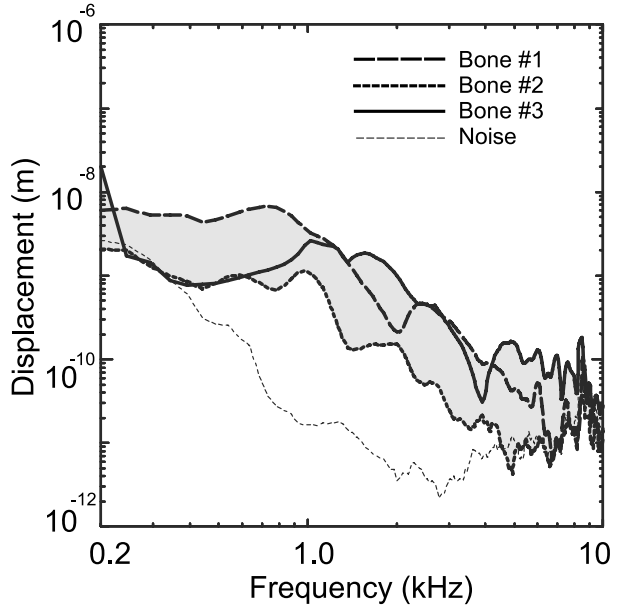

Fig. 5 Variation among the three bones for the distance of $1 \mathrm{~mm}$. The range of the variation is indicated by the gray area.

in which the measurement data are larger than the noise level.

When the distance between the vibrator coil and the magnet is $1 \mathrm{~mm}$, although the displacement amplitudes of the stapes obtained from Bones \#1, \#2 and \#3 have several peaks and notches above $6.0 \mathrm{kHz}$, they are larger than those excited by acoustical stimulation of $60 \mathrm{~dB}$ SPL in the frequency ranges of $0.2-5.0 \mathrm{kHz}$ (Bone \#1), $0.35-$ $4.5 \mathrm{kHz}$ (Bone \#2) and $0.35-10 \mathrm{kHz}$ (Bone \#3).

When the distance between the magnet and the vibrator coil is changed from 1 to $3 \mathrm{~mm}$, the displacement amplitude of the stapes exhibits a nearly downward parallel shift above $0.3 \mathrm{kHz}$ in Bone \#1 and above $0.5 \mathrm{kHz}$ in Bone \#3, except for several notches and peaks above $4.0 \mathrm{kHz}$. The mean reduction of the displacement amplitude in Bone \#1 is a factor of $3.2(10 \mathrm{~dB})$ in the frequency range of $0.3-4.0 \mathrm{kHz}$ and that in Bone \#3 is $5.6(15 \mathrm{~dB})$ in the frequency range of $0.5-4.0 \mathrm{kHz}$. By contrast, in Bone \#2, the reduction in the displacement amplitude is a factor of $5.6(15 \mathrm{~dB})$ at $1.5 \mathrm{kHz}$ and becomes $1.0(0 \mathrm{~dB})$ at $4.2 \mathrm{kHz}$.

Figure 5 shows the measurement data for the distance of $1 \mathrm{~mm}$ in Bones \#1, \#2 and \#3. The maximum difference in the displacement amplitude of the stapes between Bones \#1 and \#2, Bones \#2 and \#3, and Bones \#1 and $\# 3$ are $24 \mathrm{~dB}$ at $3.0 \mathrm{kHz}, 33 \mathrm{~dB}$ at $4.9 \mathrm{kHz}$ and $20 \mathrm{~dB}$ at $7.0 \mathrm{kHz}$, respectively. The displacement amplitude in Bone \#2 is smaller than those in Bones \#1 and \#3 over the entire measurable frequency range of $0.35-10 \mathrm{kHz}$.

\subsection{Equivalent sound pressure}

It has been reported that the displacement amplitude of the stapes increases linearly with an increase in sound pressure for stimulus levels ranging from 82 to $106 \mathrm{~dB}$ in human temporal bones ${ }^{(15)}$. This means that the displacement amplitude of the stapes excited by a hearing aid can be converted to that in terms of sound pressure level. In

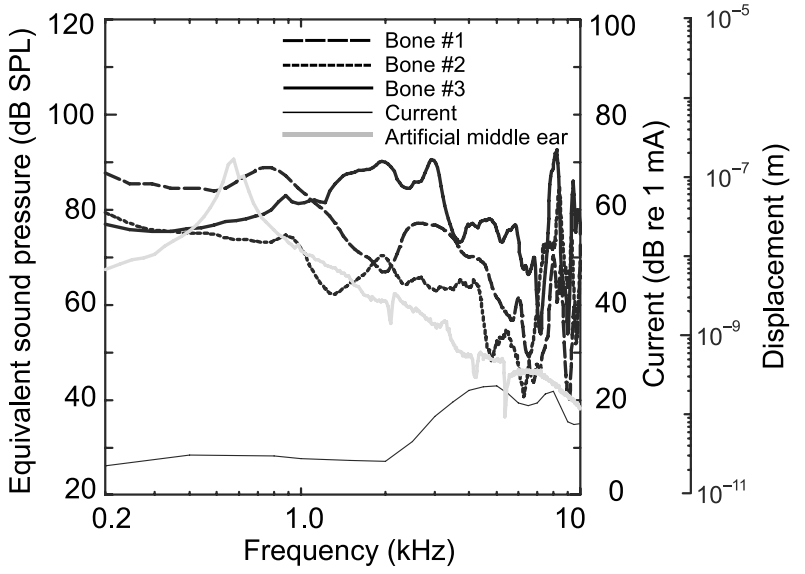

Fig. 6 The frequency responses of equivalent sound pressure for a distance of $1 \mathrm{~mm}$, the current, which flows to the driving coil when a sound pressure of $60 \mathrm{~dB}$ is applied to the microphone of the hearing aid, and the displacement amplitude at the center of the tympanic membrane of the artificial middle ear when a constant current of $10 \mathrm{~mA}$ is directly applied to the driving coil.

this study, this sound pressure is defined as "equivalent sound pressure level," $P_{E Q}$, and it is represented by

$$
P_{E Q}=60+20 \times \log \frac{D_{\text {aid }}}{D_{\text {acoust }}},
$$

where $D_{\text {aid }}$ and $D_{\text {acoust }}$ are displacement amplitudes at the posterior crus of the stapes excited by the hearing aid and by an acoustical stimulus of $60 \mathrm{~dB}$ SPL, respectively.

The frequency responses of the equivalent sound pressure generated by the hearing aid for a distance of $1 \mathrm{~mm}$ are shown in Fig. 6. In this figure, the current which flows to the driving coil (Fig. 3) and the displacement amplitude at the center of the tympanic membrane of the artificial middle ear, which is obtained when a constant current of $10 \mathrm{~mA}$ is applied to the driving coil, are also shown. This artificial middle ear was made so as to have a resonance frequency of $0.8 \mathrm{kHz}$ because Gyo et al. ${ }^{(16)}$ and Hato et al. ${ }^{(17)}$ reported that the resonance frequency of the middle ear of human temporal bones was between 0.7 and $1.0 \mathrm{kHz}$.

All curves of the equivalent sound pressure contain several notches and peaks above $6.0-10 \mathrm{kHz}$. These notches and peaks are caused by the difference in the resonance frequencies between the middle ear with and without the vibrator coil. In Bone \#1, the equivalent sound pressure is more than $80 \mathrm{~dB}$ up to $1.3 \mathrm{kHz}$ and the maximum value is $90 \mathrm{~dB}$ at $0.75 \mathrm{kHz}$. In the case of Bone \#2, the equivalent sound pressure is less than $80 \mathrm{~dB}$ over the entire frequency range of $0.2-10 \mathrm{kHz}$, except for the peak at $8.5 \mathrm{kHz}$. In Bone \#3, the equivalent sound pressure is more than $80 \mathrm{~dB}$ in the frequency range between 0.8 and $3.2 \mathrm{kHz}$. The maximum value in this frequency range is $91 \mathrm{~dB}$ at 2.0 and $3.0 \mathrm{kHz}$. 


\section{Discussion}

\subsection{Frequency responses of the stapes}

The displacement amplitude in Bone \#2 is smaller than those in Bones \#1 and \#3 over the entire measurable frequency range of $0.35-10 \mathrm{kHz}$. In this bone, it was difficult to attach the vibrator coil to the tympanic membrane because shape of the plastic plate did not correspond to that of the tympanic membrane and the vibrator coil was unstable. This unstable adherence of the vibrator coil might have caused the reduction of the excitation force as compared with results obtained from Bones \#1 and \#3.

According to theoretical analysis ${ }^{(14)}$, when the distance between the magnet and the vibrator coil is changed from 1 to $3 \mathrm{~mm}$, the decrease of the displacement amplitude is $10.4 \mathrm{~dB}$ and independent of the frequency. The mean reductions of the displacement amplitude obtained from Bone \#1 (10 dB) and Bone \#3 (15 dB) in Fig. 4 are similar to that calculated by theoretical analysis. However, it is difficult to theoretically explain the result obtained from Bone \#2, as shown in Fig. 4 (b), because the difference in the displacement amplitude decreases with an increase in the frequency. The reason for this result is also considered to be the unstableness of the vibrator coil.

As shown in Fig. 5, the maximum difference in the displacement amplitude of the stapes between Bones \#1 and \#3 extracted from the cadavers of 63- and 46-year-old males was $20 \mathrm{~dB}$ at $7.0 \mathrm{kHz}$. Goode et al. ${ }^{(17)}$ reported that the difference between the sound-induced umbo velocities measured in 64 subjects ranging in age from 10 to 79 years was less than $10 \mathrm{~dB}$. Aibara et al. ${ }^{(18)}$ measured the stapes velocities in 11 human temporal bones of subjects ranging in age from 36 to 81 years and found the variation of the displacement amplitude of the stapes to be $20 \mathrm{~dB}$. From these data, it is considered that the 20-dB difference in the displacement amplitude of the stapes between Bones \#1 and \#3 in this study was probably caused by the individual differences between the subjects.

\subsection{Equivalent sound pressure}

As shown in Fig. 6, our previous experiment using an artificial middle ear showed that when a constant current of $10 \mathrm{~mA}$ is applied to the driving coil, the displacement amplitude at the center of the tympanic membrane decreases with an increase in the frequency above the resonance frequency of $0.5 \mathrm{kHz}$, except for some notches. On the other hand, slopes of the equivalent sound pressure obtained from Bones \#1, \#2 and \#3 are not as steep as that obtained from the artificial middle ear in the frequency range between $2.0 \mathrm{kHz}$ and $5.0 \mathrm{kHz}$. As shown in Figs. 3 and 6 , the frequency response of the current, which flows to the driving coil, increases with an increase in the frequency from $2.0 \mathrm{kHz}$ and the maximum current at $5.0 \mathrm{kHz}$ is $15 \mathrm{~dB}$ larger than that at $2.0 \mathrm{kHz}$. According to the theoretical analysis in our previous study ${ }^{(14)}$, the excitation force increases linearly with an increase in the current, which results in the increase of the equivalent sound pressure obtained from the three bones.

\subsection{Comparison with other transducers}

Generally, hearing aids must amplify the acoustical input signal so as to acquire a sufficient acoustical gain needed by the hearing aid wearer. Acoustical gain is defined as the ratio of sound pressure level generated by a hearing aid to that received by its microphone. Figure 7 shows the frequency characteristics of the acoustical gain calculated on the basis of the results in Fig. 6. In this figure, the acoustical gains of other hearing aids developed in the past are also shown. In the hearing aid herein presented, the acoustical gain increases with an increase in the frequency and its maximum value is $31 \mathrm{~dB}$ at 2.0 and $3.0 \mathrm{kHz}$.

A transducer used in the Vibrant Soundbridge ${ }^{\circledR}$ (Hough et al. 2002), mentioned in the introduction, is called Floating Mass Transducer ${ }^{\mathrm{TM}}$. This transducer is coupled to the incus and excites the ossicles directly by a magnet in a titanium container. The acoustical gain of this hearing aid is larger than that of ours in the frequency range between 3.0 and $5.5 \mathrm{kHz}^{(3)}$. However, to implant the transducer of the hearing aid, the tympanic cavity must be exposed by surgery. Wang et al. ${ }^{(11)}$ recently developed a small multi-layer piezoelectric transducer for use in a hearing aid. This transducer is cemented between the medial wall of the attic of the middle ear and the body of the incus. Contrary to the acoustical gain of our hearing aid, that of the hearing aid developed by Wang et al. continues to increase with an increase in the frequency from $3.0 \mathrm{kHz}$. However, this hearing aid also must be implanted by invasive surgery. This disadvantage has prevented these implantable hearing aids from being more widely used.

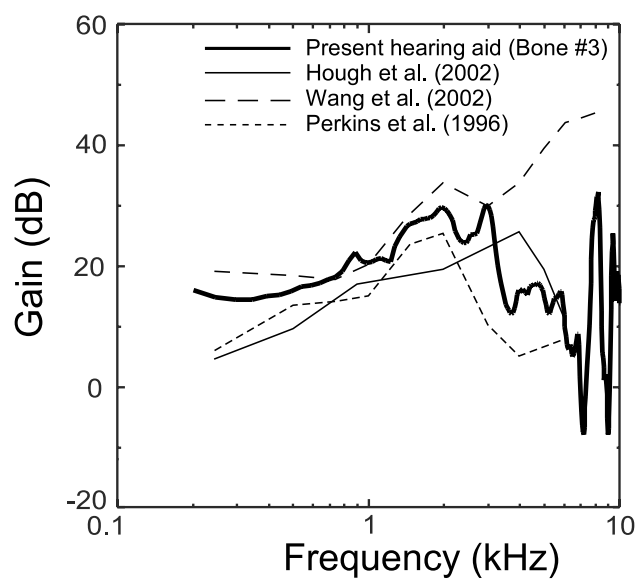

Fig. 7 Frequency characteristics of the acoustical gain of our hearing aid and those of other hearing aids developed in the past. The acoustical gain is calculated by subtracting the input sound pressure of $60 \mathrm{~dB}$ from the equivalent sound pressure on the basis of the results in Fig. 6 . 
The non-implantable electromagnetic hearing aid developed by Perkins et al. ${ }^{(13)}$ suggested another approach to holding the magnet on the tympanic membrane by a silicone disk similar to a contact lens, namely Earlens ${ }^{\circledR}$. However, the acoustical gain of this hearing aid is lower than that of ours over the frequency range between 0.25 and $6.0 \mathrm{kHz}$.

The non-implantable hearing aid developed in the present study is able to obtain larger acoustical gain in contrast with the non-implantable hearing aid developed by Perkins et al. ${ }^{(13)}$ One reason is that the induced current generated in the transducer used in our hearing aid increases linearly with an increase in the frequency, resulting in an increase of the excitation force. Another reason is that our hearing aid uses a lightweight-vibrator coil weighing $18.3 \mathrm{mg}$, while the weight of Earlens ${ }^{\circledR}$ is approximately $40 \mathrm{mg}$. A vibrator coil with a small mass is essential for efficiency of the electromagnetic transducer at high frequencies. These advantages, i.e., the increase of the excitation force and the lightweight coil make it possible to achieve high acoustical gain.

However, the acoustical gain at high frequencies was not as great as that obtained from the hearing aid developed by Wang et al. ${ }^{(11)}$ One possible reason is the difference in the vibration modes of the stapes caused by the hearing aid and the acoustical stimulation. Hato et al. ${ }^{(17)}$ reported that the stapes motion is a combination of piston-like motion and rotational motion and that the stapes motion is complicated, especially at high frequencies. As the displacement amplitude was measured at only one point of the stapes in this study, it is probably affected by such vibration modes of the stapes, leading to a small displacement amplitude, resulting in a decrease of the acoustical gain. However, the decrease in the displacement amplitude at the posterior crus of the stapes measured in this study does not represent the decrease in the sound transmission to the inner ear because the volume displacement of the stapes footplate is inestimable by this method. Rather, hearing should be estimated by physiological responses, e.g., cochlear microphonic (CM) since it arises from the sum of the electrical potentials in hair cells in the cochlea. The CM measurement using anesthetized guinea pigs in our previous study showed that the transducer generated a high excitation force even at high frequencies. This suggests that although the acoustical gain of our hearing aid calculated from the displacement amplitude of the stapes is smaller than that of the hearing aid developed by Wang et al. at high frequencies, the newly developed hearing aid is applicable to patients with hearing loss even at high frequencies.

\section{Conclusions}

The best result of experiments using three bones indicates that the newly developed hearing aid herein pre- sented can generate an excitation force of more than $80 \mathrm{~dB}$ SPL in terms of sound pressure at frequencies between 0.8 and $3.2 \mathrm{kHz}$.

\section{Acknowledgements}

The authors are grateful to Prof. Emeritus William T. Peake at Eaton-Peabody Laboratory, Massachusetts Eye and Ear Infirmary, Boston, MA, U.S.A. for their helpful comments and skillful technical assistance. This work was supported by a Grant-in-Aid for Scientific Research (No. 17700440 ) from the Ministry of Education, Culture, Sports, Science and Technology of Japan, by a Health and Labour Science Research Grant from the Ministry of Health, Labour and Welfare of Japan and by the 21st Century COE Program Special Research Grant of the "Future Medical Engineering Based on Bio-nanotechnology".

\section{References}

( 1 ) Suzuki, J., Kodera, K., Nagao, K. and Yabe, T., Partially Implantable Piezoelectric Middle Ear Hearing Device, Otolaryngol. Clin. North Am., Vol.28 (1995), pp.99-106.

(2) Gyo, K., Saiki, T. and Yanagihara, N., Implantable Hearing Aid Using a Piezoelectric Ossicular Vibrator: A Speech Audiometric Study, Audiology, Vol.35 (1996), pp.271-276.

( 3 ) Hough, M.D., Dyer, R.K.J., Matthews, P. and Wood, M.W., Early Clinical Results: SOUNDTEC Implantable Hearing Device Phase 2 Study, Laryngoscope, Vol.111 (2002), pp.1-8.

(4) Heide, J., Tatge, G. and Sander, T., Development of a Semi-Implantable Hearing Aid, Adv. Audiol., Vol.4 (1988), pp.42-43.

( 5 ) Dumon, T., Zennaro, O. and Aran, J., Piezoelectric Middle Ear Implant: Experimental Results [Abstract 35], International Symposium on Electronic Implants in Otology, Orlando, FL, November, (1993).

( 6 ) Tos, M., Salomon, G. and Bonding, P., Implantation of Electromagnetic Ossicular Replacement Device, Ear Nose Throat J., Vol.73 (1994), pp.92-103.

( 7 ) Gan, R.Z., Dormer, K.J., Ball, G.R. and Dietz, T.G., Implantable Hearing Device Performance Measured by Laser Doppler Interferometry, Ear Nose Throat J., Vol.76 (1997), pp.297-309.

( 8 ) Goode, R.L., Current Status and Future of Implantable Electromagnetic Hearing Aids, Otolaryngol. Clin. North Am., Vol.28 (1995), pp.141-146.

(9) Goode, R.L., Rosenbaum, M.A. and Maniglia, A.J., The History and Development of the Implantable Hearing Aid, Otolaryngol. Clin. North Am., Vol.28 (1995), pp.1-16.

(10) Frederickson, J.M., Coticchia, J.M. and Khosla, S., Ongoing Investigation into an Implantable Electromagnetic Hearing Aid for Moderate to Severe Sensorineural Hearing Loss, Otolaryngol. Clin. North Am., Vol.28 (1995), pp.107-120.

(11) Wang, Z.G., Abel, E.W., Mills, R.P. and Liu, Y., Assessment of Multi-Layer Piezoelectric Actuator Tech- 
nology for Middle-Ear Implants, Mechatronics, Vol.12 (2002), pp.3-17.

(12) Park, S., Lee, K.C., Song, B.S., Cho, J.H., Kim, M.N. and Lee, S.H., Microvibration Transducer Using Silicone Elastic Body for an Implantable Middle Ear Hearing Aid, Mechatronics, Vol.12 (2002), pp.1173-1184.

(13) Perkins, R., Earlens Tympanic Contact Transducer: A New Method of Sound Transduction to the Human Ear, Otolaryngol. Head Neck Surg., Vol.114 (1996), pp.720-728.

(14) Hamanishi, S., Koike, T., Matsuki, H. and Wada, H., A New Electromagnetic Hearing Aid Using Lightweight Coils to Vibrate the Ossicles, IEEE Trans. Mag., Vol.40 (2004), pp.3387-3393.

(15) Merchant, S.N., Ravicz, M.E. and Rosowski, J.J., Acoustic Input Impedance of the Stapes and Cochlea in Human Temporal Bones, Hear. Res., Vol.97 (1996), pp.30-45.

(16) Gyo, K., Arimoto, H. and Goode, R.L., Measurement of the Ossicular Vibration Ratio in Human Temporal Bones by Use of a Video Measuring System, Acta Otolaryngol., Vol.103 (1987), pp.87-95.

(17) Hato, N., Stenfelt, S. and Goode, R.L., ThreeDimensional Stapes Footplate Motion in Human Temporal Bones, Audiology \& Neuro-Otology, Vol.8 (2003), pp.140-152.

(18) Goode, R.L., Ball, G., Nishihara, S. and Nakamura, K., Laser Doppler Vibrometer (LDV) - A New Clinical Tool for the Otologist, A.J. Otol., Vol.17 (1996), pp.813-822.

(19) Aibara, R., Welsh, J.T., Puria, S. and Goode, R.L., Human Middle-Ear Sound Transfer Function and Cochlear Input Impedance, Hear. Res., Vol.152 (2001), pp.100-109. 